\title{
Far East Scarlet-like Fever Masquerading as Adult-onset Kawasaki Disease
}

\author{
Kazuki Ocho ${ }^{1}$, Masaya Iwamuro ${ }^{1}$, Kou Hasegawa ${ }^{1}$, Hideharu Hagiya ${ }^{2}$, Kammei Rai ${ }^{1}$, \\ Tetsuya Yumoto ${ }^{3}$ and Fumio Otsuka ${ }^{1}$
}

\begin{abstract}
:
A previously healthy 31-year-old man was referred to us with refractory septic shock accompanied by bilateral conjunctival congestion and erythema of his right lower limb. Nine days after admission, he had bilateral desquamation of the fingertips, and his presentation satisfied the criteria for Kawasaki disease. A serological examination was positive for Yersinia pseudotuberculosis, and he was diagnosed with Far East scarletlike fever (FESLF). Interestingly, his 11-month-old baby boy had similar symptoms around the same time, indicating the intrafamilial transmission of the pathogen. We should consider FESLF when we encounter a familial occurrence of systemic manifestations of Kawasaki disease.
\end{abstract}

Key words: Yersinia pseudotuberculosis, adult-onset Kawasaki disease, domestic infection, Far East scarletlike fever, sepsis

(Intern Med 57: 437-440, 2018)

(DOI: 10.2169/internalmedicine.9250-17)

\section{Introduction}

Yersinia pseudotuberculosis is a gram-negative rod that a member of the Enterobacteriaceae family. The organism usually causes gastroenteritis in humans, is more common in Europe than in Asia (1), and affects children more frequently than adults (2). In countries in the Far East such as Japan and eastern Russia, cases of $Y$. pseudotuberculosis infection with systemic manifestations have been reported $(2,3)$. Its major symptoms include a high fever, generalized rash, strawberry tongue, conjunctival congestion, and renal failure (4), mimicking the clinical presentation of Kawasaki disease (5). This disease is called Far East scarletlike fever (FESLF), scarlatinoid fever, or Izumi fever in Japan (2). The organism is transmitted orally via the ingestion of contaminated food or well water $(6,7)$, and contact with vectors (8). Thus, outbreaks of this Kawasaki disease-like infectious disease have occasionally been described (9).

We encountered an adult patient with FESLF who had a systemic Y. pseudotuberculosis infection, and who fulfilled the diagnostic criteria for Kawasaki disease. Interestingly, his 11-month-old baby boy concurrently suffered from symptoms similar to those seen in Kawasaki disease.

\section{Case Report}

A 31-year-old man was admitted to a hospital with high fever and a sore throat in late February. The patient was previously healthy and did not take any medications. He lived with his wife and 11-month-old baby boy and had started keeping a dog one month before the onset of his symptoms. Three weeks previously, his son suffered from high fever, strawberry tongue, and desquamation of the fingertips, which resolved within a few days without any treatment.

On admission, his body temperature was elevated to $39^{\circ} \mathrm{C}$. A physical examination revealed a swollen and reddened pharynx and erythema of the trunk and right thigh. The results of streptococcal antibody tests including antistreptolysin $\mathrm{O}$ and anti-streptokinase antibodies were negative. Ampicillin/sulbactam (3 g, 8-hourly) was empirically administered based on the suspicion of a bacterial infection

\footnotetext{
${ }^{1}$ Department of General Medicine, Okayama University Graduate School of Medicine, Dentistry, and Pharmaceutical Sciences, Japan, ${ }^{2}$ Division of Infection Control and Prevention, Osaka University Hospital, Japan and ${ }^{3}$ Department of Emergency Medicine, Okayama University Graduate School of Medicine, Dentistry, and Pharmaceutical Sciences, Japan

Received: March 27, 2017; Accepted: June 4, 2017; Advance Publication by J-STAGE: November 1, 2017

Correspondence to Dr. Kazuki Ocho, m03009ko@jichi.ac.jp
} 


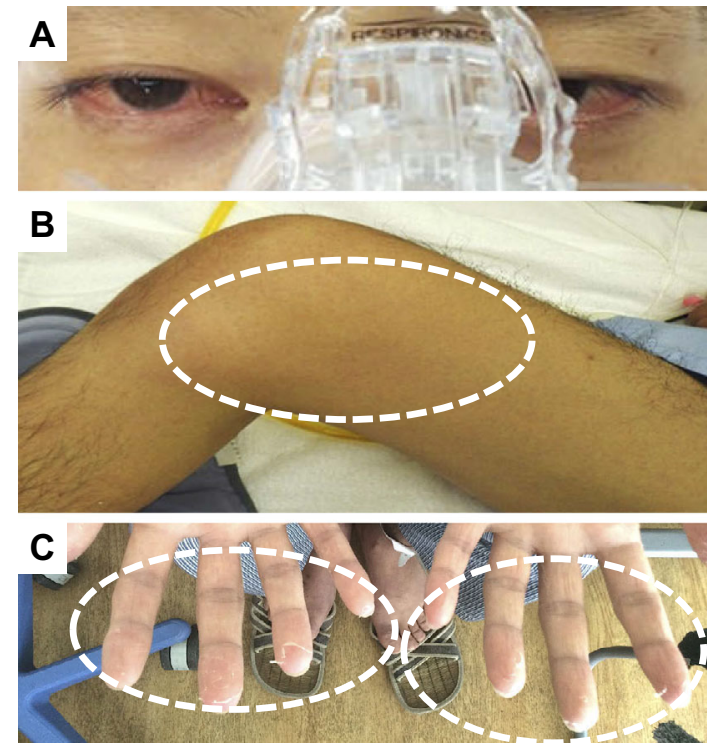

Figure. A photograph of the patient. Bilateral conjunctival congestion (A), multiple light erythema at the right lower limb (B), and bilateral stripped fingertips on the 9th day of admis$\operatorname{sion}(\mathrm{C})$.

of the upper respiratory tract. However, the patient went into septic shock, requiring noradrenalin support, and was referred to our hospital for further investigation and treatment. There were no gastrointestinal symptoms, including abdominal pain and diarrhea.

On arrival, the patient was well oriented, and his vital signs were as follows: blood pressure, 112/54 $\mathrm{mmHg}$ on continuous infusion of noradrenaline $(0.19 \mu \mathrm{g} / \mathrm{kg} / \mathrm{min})$; heart rate, 110 beats/min; respiratory rate, 24 breaths/min; oxygen saturation, $96 \%$ (on $3 \mathrm{~L} / \mathrm{min}$ of oxygen); and body temperature, $37.3^{\circ} \mathrm{C}$. Both conjunctivae were congested (Figure A), and multiple areas of erythema were seen on his right lower limb (Figure B). Laboratory findings demonstrated an increased white blood cell count $(27,650 / \mu \mathrm{L})$, an elevated erythrocyte sedimentation rate $(79 \mathrm{~mm} / \mathrm{h})$, elevated levels of C-reactive protein $(27.77 \mathrm{mg} / \mathrm{dL})$, total and direct bilirubin $(5.88 \mathrm{mg} / \mathrm{dL}$ and $4.09 \mathrm{mg} / \mathrm{dL}$ respectively), serum ferritin (911.7 ng/mL), soluble interleukin-2 receptor $(3,837 \mathrm{U} / \mathrm{mL})$, brain natriuretic peptide $(1,850.3 \mathrm{pg} / \mathrm{mL})$, and procalcitonin $(2.53 \mathrm{ng} / \mathrm{mL})$, and decreased serum levels of total protein $(4.5 \mathrm{~g} / \mathrm{dL})$ and albumin $(1.7 \mathrm{~g} / \mathrm{dL})$. His platelet count on admission was normal $(269,000 / \mu \mathrm{L})$. Blood, urine, and cerebrospinal fluid cultures were unremarkable throughout the admission. Contrast-enhanced computed tomography (CT) scanning revealed bilaterally enlarged posterior cervical lymph nodes, pulmonary congestion, and mild splenomegaly. No lymphadenopathy was observed at the abdominal and ileocecal lesions.

Treatment was initiated for septic shock of unknown cause in the intensive care unit with IV meropenem and gamma-globulin ( $5 \mathrm{~g} /$ day for 3 days). On the second day of admission, we changed the antibiotic to a combination of levofloxacin (500 mg every 24 hours) with clindamycin
(600 mg every 8 hours), because his fever had not resolved. On the fifth day of admission, we added minocycline (100 mg, every 12 hours), when a Leptospira infection was suspected. The fever and his general condition gradually improved, and the patient was moved to the general ward on the ninth day after admission. On that day, we noticed a bilateral desquamation of his fingertips (Figure C). His clinical condition met the clinical criteria for Kawasaki disease, which are as follows: i) fever persisting for at least 5 days; ii) bilateral, painless bulbar conjunctival congestion without exudate; iii) cervical lymphadenopathy; iv) polymorphous exanthema; and v) changes in the extremities such as membranous desquamation of the fingertips. The number of peripheral blood platelets was progressively increasing during the recovery period (maximum number: $869,000 / \mu \mathrm{L}$ ), which was also compatible with Kawasaki disease. Coronary CT angiography on the 16th day after admission revealed no lesions, including aneurysm formation. Cardiac ultrasonography performed at 2 months after admission was unremarkable. Paired serum samples to anti-leptospiral antibodies were negative on the $3 \mathrm{rd}$ and 17 th days after admission. He was discharged 20 days after admission and showed no signs of recurrence.

We considered streptococcal or staphylococcal toxic shock syndromes as the primary differential diagnoses. However, he did not meet the criteria for either the CDC 2010 Streptococcal Toxic Shock Syndrome Case Definition or the CDC 2011 Toxic Shock Syndrome Case Definition. Furthermore, the increase in the platelet count during the recovery period could not be accounted for by these diseases. Considering the Kawasaki disease-like manifestations in an adult patient, we suspected FESLF as the most probable cause (10). Paired serum samples were negative for anti-Y. pseudotuberculosis mitogen (YPM) antibodies. However, an agglutination reaction test of a blood sample obtained on the 4th day of admission was positive for $Y$. pseudotuberculosis antibody (YP2a) positive (1:160). Consequently, we diagnosed the patient with FESLF caused by $Y$. pseudotuberculosis.

\section{Discussion}

We presented a rare case of FESLF in an adult Japanese man. The clinical manifestations of the patient satisfied the criteria for Kawasaki disease, but the final diagnosis of FESLF was successfully made based on the elevation of $Y$. pseudotuberculosis specific antibody levels. Y. pseudotuberculosis septicemia in adults typically affects patients with hepatic and hematologic diseases (11) and is rare in healthy individuals. It is interesting to note that prior to the onset of the disease, his son had suffered from similar symptoms, suggesting the domestic transmission of the pathogen.

Systemic Y. pseudotuberculosis infection generally develops in childhood, causing fever, rash, abdominal symptoms (diarrhea and abdominal pain), eye congestion, strawberry tongue, and lymphadenopathy in the acute phase. Patients with the disease can be complicated with renal failure, 
erythema nodosum, and desquamation at the distal portion of the extremities in the subacute phase (9). However, the disease spectrum is considerably wide and the clinical course varies from patient to patient. In our case, it was noteworthy that the patient met the diagnostic criteria for Kawasaki disease (adult-onset, and the absence of gastrointestinal symptoms).

Patients with FESLF present with similar symptoms as Kawasaki disease. On admission, the patient had a fever, hypotension, conjunctival congestion, multiple erythema, and icterus. Accordingly, the differential diagnoses included toxic shock syndromes and severe leptospiral infection. However, the results of investigations were not suggestive of these other infectious etiologies. Instead, the emergence of fingertip stripping in the convalescent stage reminded us of $Y$. pseudotuberculosis infection and was confirmed by a positive agglutination test result for the $Y$. pseudotuberculosis specific antibody.

There is a controversy regarding the relationship between Kawasaki disease and $Y$. pseudotuberculosis infection. In a Japanese retrospective cohort study involving 452 pediatric patients with Kawasaki disease, the stool cultures of 42 patients $(9.3 \%)$ tested positive for $Y$. pseudotuberculosis (12). The Yersinia-positive patients frequently had coronary artery lesions $(52.4 \%$ vs. $31.8 \%, \mathrm{p}=0.001)$ and presented with a refractory course $(36.1 \%$ vs. $16.0 \%, \mathrm{p}=0.004)$, in comparison to the Yersinia-negative patients. These findings indicate the relevance of $Y$. pseudotuberculosis infection in severe forms of Kawasaki disease. Another hypothesis about the relationship between Kawasaki disease and Y. pseudotuberculosis infection is that there may be a common antigen between $Y$. pseudotuberculosis and the unknown organism(s) that cause Kawasaki disease (13). Irrespective of the relationship between the two diseases, physicians should remember to evaluate Kawasaki disease for $Y$. pseudotuberculosis infection and vice versa.

It was also noteworthy that the patient's 11-month-old baby boy, who was living with him, had similar symptoms around the same time. This chronologically relevant onset of similar symptoms in family members suggested an infectious etiology, although $Y$. pseudotuberculosis infection was not detected in the boy. Among the 164 Y. pseudotuberculosis infections that have previously been reported in Japan, 22 cases (11 families) occurred as intrafamilial infections (9). Thus, we assume that this case could also represent a case of domestic $Y$. pseudotuberculosis infection. In such cases, although the patient in the present case denied its use, contaminated well water is a probable source (9). Another possibility is that his dog was the source of infection, since domestic animals can be carriers of $Y$. pseudotuberculosis (14). Previous cases have reported the transmission of Yersinia enterocolitica infection from household dogs to humans $(15,16)$. However, the source remained unknown in this case. Since the first report of Izumi fever in Kanazawa in 1927, FESLF has been widely reported in Japan without a geographical bias $(9,14,17)$. Thus, we should consider this disease in patients who show Kawasaki disease-like symptoms, especially when intrafamilial transmission is suspected.

The refractory shock observed in this patient could be attributable to a toxigenic reaction induced by YPM. This exotoxin has superantigen activity that causes toxic shock in healthy mice, but not in T-cell deficient mice with severe combined immunodeficiency (18). The injection of YPM also causes the activation of Vbeta8 (+) T cells [a type of natural killer (NK)-T cell], which relocates from the peripheral blood to the liver (18). These in vitro findings are suggestive of YPM-associated toxic shock in patients with $Y$. pseudotuberculosis infection, in relation to the activation of Vbeta8 (+) $\mathrm{T}$ cells (18). The negative result for the antiYPM antibodies in our patient may be a false-negative since the sensitivity of the test in a systemic infection is reported to be $61 \%$ (19).

Due to its rarity, the optimal treatment of this disease has not yet been established. Y. pseudotuberculosis is generally susceptible to a wide range of antibiotics (20), and thirdgeneration cephalosporins or fluoroquinolones are generally given (21). Based on the management of Yersinia enterocolitica septicemia, it is recommended that treatment be administered for a period of 3 weeks (21).

In summary, we encountered the case of an adult patient with systemic $Y$. pseudotuberculosis infection, or FESLF, in which the clinical manifestations resembled those of Kawasaki disease. The preceding onset of similar symptoms in his 11-month-old baby boy suggested the intrafamilial transmission of the pathogen.

The authors state that they have no Conflict of Interest (COI).

\section{Acknowledgement}

We express our sincere thanks to Dr. Hiroshi Nakajima (Okayama Prefecture Environment Health Center) for the measurement of the titer of serum hemagglutinin to Y. pseudotuberculosis, and Dr. Jun Abe (National Center for Child Health and Development) for the measurement of the anti-YPM antibodies.

\section{References}

1. Ostroff S. Yersinia as an emerging infection: epidemiologic aspects of Yersiniosis. Contributions to microbiology and immunology 13: 5-10, 1995.

2. Sato K, Ouchi K, Taki M. Yersinia pseudotuberculosis infection in children, resembling Izumi fever and Kawasaki syndrome. Pediatr Infect Dis J 2: 123-126, 1983.

3. Amphlett A. Far East scarlet-like fever: a review of the epidemiology, symptomatology, and role of superantigenic toxin: Yersinia pseudotuberculosis-derived mitogen A. Open Forum Infect Dis 3: ofv202, 2015.

4. Inoue $\mathrm{M}$, Nakashima $\mathrm{H}$, Ueba $\mathrm{O}$, et al. Community outbreak of Yersinia pseudotuberculosis. Microbiol Immunol 28: 883-891, 1984.

5. Horinouchi T, Nozu K, Hamahira K, et al. Yersinia pseudotuberculosis infection in Kawasaki disease and its clinical characteristics. BMC Pediatrics 15: 177, 2015.

6. Nuorti JP, Niskanen T, Hallanvuo S, et al. A widespread outbreak 
of Yersinia pseudotuberculosis 0:3 infection from iceberg lettuce. J Infect Dis 189: 766-774, 2004.

7. Rimhanen-Finne R, Niskanen T, Hallanvuo S, et al. Yersinia pseudotuberculosis causing a large outbreak associated with carrots in Finland, 2006. Epidemiol Infect 137: 342-347, 2009.

8. Slee KJ, Skilbeck NW. Epidemiology of Yersinia pseudotuberculosis and $Y$. enterocolitica infections in sheep in Australia. J Clin Microbiol 30: 712, 1992.

9. Sato K. Clinical findings and epidemiology of Yersinia pseudotuberculosis infection, especially concerned with Izumi fever. Kansenshogaku Zassi (J Jpn Assoc Infect Dis) 61: 746-762, 1987 (in Japanese).

10. Vincent P, Salo E, Skumik M, Fukushima H, Simonet M. Similarities of Kawasaki disease and Yersinia pseudotuberculosis infection epidemiology. Pediatr Infect Dis J 26: 629-631, 2007.

11. Marlon A, Gentry L, Merigan TC. Septicemia with Pasteurella pseudotuberculosis and liver disease. Arch Intern Med 127: 947949, 1971.

12. Tahara M, Baba K, Waki K, Arakaki Y. Analysis of Kawasaki disease showing elevated antibody titres of Yersinia pseudotuberculosis. Acta Paediatr 95: 1661-1664, 2006.

13. Tanaka M, Takimoto T, Hongo T. Yersinia pseudotuberculosis infection. Rinsho Byori (Jpn J Clin Pathol) 33: 598-605, 1985 (in Japanese).

14. Tsubokura M, Otsuki K, Sato K. Distribution of Yersinia pseudotuberculosis in Japan and epidemiology of human infection. Kansenshogaku Zassi (J Jpn Assoc Infect Dis) 61: 737-745, 1987 (in Japanese)
15. Wilson HD, McCormick JB, Fleeley JC. Yersinia enterocolitica infection in a 4-month-old infant associated with infection in household dogs. J Pediatr 89: 767-769, 1976.

16. Gutman LT, Ottesen EA, Quan TJ, Noce PS, Katz SL. An inter familial outbreak of Yersinia enterocolitica enteritis. N Engl J Med 288: 1372-1377, 1973.

17. Toyokawa Y, Ohmoto Y, Akiyama T. Large scale outbreak of Yersinia pseudotuberculosis serotype 5a infection at Noheji-machi in Aomori Prefecture. Kansenshogaku Zassi (J Jpn Assoc Infect Dis) 67: 36-44, 1992 (in Japanese, Abstract in English).

18. Kano $\mathrm{H}$, Ito $\mathrm{Y}$, Matsuoka $\mathrm{K}$, et al. Critical role of $\mathrm{T}$ cell migration in bacterial superantigen-mediated shock in mice. Clin Immunol 110: 159-171, 2004.

19. Abe J, Onimaru M, Matsumoto S, et al. Clinical role for a superantigen in Yersinia pseudotuberculosis infection. J Clin Invest 99: 1823-1830, 1997.

20. Bonardi S, Bruinni I, D'Incau M, et al. Detection, seroprevalence and antimicrobial resistance of Yersinia enterocolitica and Yersinia pseudotuberculosis in pig tonsils in Northern Italy. Int J Food Microbiol 235: 125-132, 2016.

21. Gayraud M, Scavizzi MR, Mollaret HH, Guillevin L, Hornstein MJ. Antibiotic treatment of Yersinia enterocolitica septicemia: a retrospective review of 43 cases. Clin Infect Dis 17: 405-410, 1993.

The Internal Medicine is an Open Access article distributed under the Creative Commons Attribution-NonCommercial-NoDerivatives 4.0 International License. To view the details of this license, please visit (https://creativecommons.org/licenses/ by-nc-nd/4.0/).

\footnotetext{
(C) 2018 The Japanese Society of Internal Medicine
} Intern Med 57: 437-440, 2018 